\title{
Temperature-Independent High-Speed Distributed Voltage Measurement using Intensiometric FBG Interrogation
}

\author{
Grzegorz Fusiek, Philip Orr, Paweł Niewczas \\ Institute for Energy and Environment, \\ Department of Electronic and Electrical Engineering, University of Strathclyde \\ Glasgow, UK \\ g.fusiek@strath.ac.uk
}

\begin{abstract}
In this paper, we demonstrate through simulation a low-cost, high-speed optical voltage sensing system with a multiplexing capability. The scheme utilizes transducers based on a hybrid piezoelectric/optical construction, whereby the optical power reflected from dual-peak fiber Bragg gratings is modulated in response to an excitation voltage and monitored by photodetection while remaining robust to fluctuations in optical power and temperature. A dedicated solid-state interrogation system incorporating passive wavelength division multiplexing allows for high-speed measurement over the ac/dc voltage sensor array without the use of expensive components, such as tunable filters or lasers.
\end{abstract}

Keywords - optical voltage sensors, fiber Bragg grating sensors, piezoelectric transducer, intensiometric detection system.

\section{INTRODUCTION}

The growing demand for electrical energy production, transmission and distribution, due to the increased power consumption needs of industry and domestic customers, is causing existing grid infrastructure to approach inherent performance limits. To address this, the electrical power industry has, in recent years, focused on the development of smart grid technology to enable increased visibility, feedback, and control of grid operations, thus improving its efficiency, reliability and cost-effectiveness. The requirement that the smart grid should adapt immediately to real-time demand fluctuations requires a high level of grid visibility that can only be facilitated by appropriate sensing and communication systems. Power quality must also be monitored due to the increased complexity in the grid. For example, the requirements of voltage distortion monitoring and voltage and power quality monitoring, contrasted with the difficulty of voltage harmonic transfer through instrument voltage transformers have been identified as monitoring problems in medium voltage (MV) and high voltage (HV) grid systems [1], [3]. Furthermore, the high bandwidth required for smart grid communication links presents another key challenge [4].

Fiber optic technology has the potential to address many of the issues en route to the smart grid, facilitated by both the inherent dielectric nature of fiber optic sensors and the use of optical fiber as an excellent communication medium [3], [4].

Research presented in this paper was carried out within the European Metrology Research Programme (EMPR). The EMRP is jointly funded by the EMRP participating countries within EURAMET and the European Union.
Previously, we have proposed a range of hybrid voltage and current sensors based on fiber Bragg grating (FBG) technology for use in such applications as remote condition monitoring of electrical submersible pumps [5], [6], aero-electrical systems [7], and electrical network protection systems [8].

In this paper, we propose and demonstrate through simulation an optical voltage sensor array based on piezoelectric components and FBG sensors which, when combined with an intensiometric detection scheme and passive wavelength division multiplexing (WDM) filters, allow for distributed ac and dc voltage monitoring over extended distances. The system features robust, low-cost components and facilitates high sensor interrogation speed.

\section{VOLTAGE SENSOR DESIGN}

The previously proposed optical hybrid voltage sensor exploited a piezoelectric transducer with two electrodes and an FBG sensor [5]. A voltage applied across the transducer produced a relative elongation (strain) which was imparted to and measured by the FBG. Since the wavelength of an FBG changes with both strain and temperature, a suitable temperature compensation method must be implemented to provide an accurate strain/voltage measurement. This is usually realized by using an additional temperature sensing FBG that is free from mechanical strain and located in proximity of the strain measuring FBG. The technique also requires information about the spectral position of the FBG peaks and cannot be realized without relatively expensive interrogators employing tunable filters or lasers [9].

To limit the number of FBGs required for strain/voltage measurements with a decoupled temperature-dependent wavelength shift, the use of an FBG with a dual peak and intensiomentric detection scheme are considered. In the proposed approach, the FBG is twice the length of the piezoelectric element, as shown in Figure 1. One sensor electrode adjacent to the piezoelectric transducer is extended such that its length is equal to the length of the piezoelectric material. The coefficient of thermal expansion (CTE) of the piezoelectric material and the extended electrode should be carefully selected such that equal thermal strain is produced in both materials. For example invar can be used as materials for 
the extended temperature compensating electrode as their thermal expansion coefficients closely match those of the majority of ceramic piezoelectrics.

In order to produce a dual-peak reflection spectrum, the FBG attachment process is divided into two stages: firstly, half of an FBG is attached to the extended electrode using an epoxy adhesive. When the epoxy is cured, the other half of the FBG is prestressed to split the single Bragg reflection, and thereafter epoxied to the piezoelectric material. The extended electrode may then be attached to a rigid conductor supporting the sensor structure, while the second electrode can be attached to a flexible conductor allowing for unrestricted expansion and contraction of the piezoelectric element and the extended electrode.

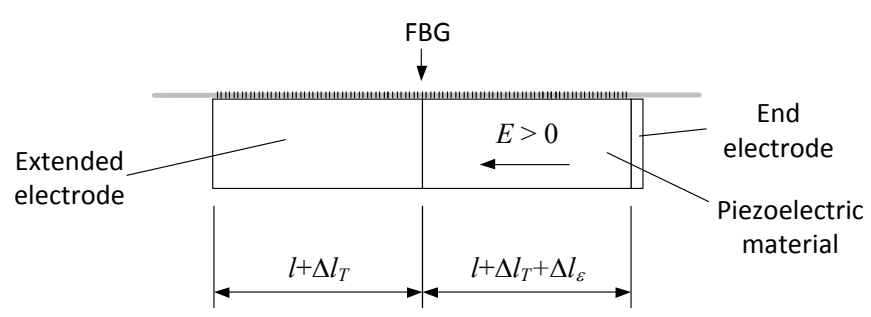

Figure 1. Conceptual voltage sensor construction.

Provided there is no mechanical stress in the piezoelectric material, the strain (i.e. relative elongation $\Delta l_{\varepsilon} l$ ) induced by an external electric field is given by

$$
\varepsilon=d \cdot E=d \cdot \frac{V}{l}
$$

where $\mathrm{d}$ is the longitudinal piezoelectric charge constant, $\mathrm{E}$ is the electric field, $V$ is the voltage applied across the piezoelectric material and $l$ is the length of the material [5], [6].

Thermal strain, (i.e. relative elongation $\Delta l_{T} / l$ ), produced in both materials due to a temperature change, $\Delta T$, can be expressed by

$$
\varepsilon_{\text {thermal }}=C T E \cdot \Delta T
$$

A relative change in the FBG peak wavelength, $\Delta \lambda_{B} / \lambda_{B}$, due to a change in strain, $\Delta \varepsilon$, and temperature, $\Delta T$, can be expressed by

$$
\frac{\Delta \lambda_{B}}{\lambda_{B}}=C_{\varepsilon} \cdot \Delta \varepsilon+C_{T} \cdot \Delta T=C_{\varepsilon} \cdot\left(\varepsilon+\varepsilon_{\text {thermal }}\right)+C_{T} \cdot \Delta T
$$

where $C_{\varepsilon}$ and $C_{T}$ are the strain and temperature sensitivities, and $\Delta \varepsilon$ denotes the sum of the mechanically and thermally induced strains.

A dual-peak FBG can be considered as a combination of two overlapping single-peak FBGs. As shown in Figure 2, when such gratings are illuminated by a broadband light source, the optical power detected by a photodetector is equal to the integrated optical power over the reflected spectrum. The spacing between FBGs, and hence, the degree to which they overlap, determines the total reflected optical power incident on the photodetector. Provided that half of the FBG attached to the extended electrode has a center wavelength at $\lambda_{1}$, and the section of FBG attached to the piezoelectric material at $\lambda_{1}$ ', the application of positive or negative voltage across the piezoelectric material results in the $\lambda_{1}$ ' peak shifting with respect to the $\lambda_{1}$ peak and its nominal position (as shown in Figure 2), translating to a modulation of the reflected optical power.

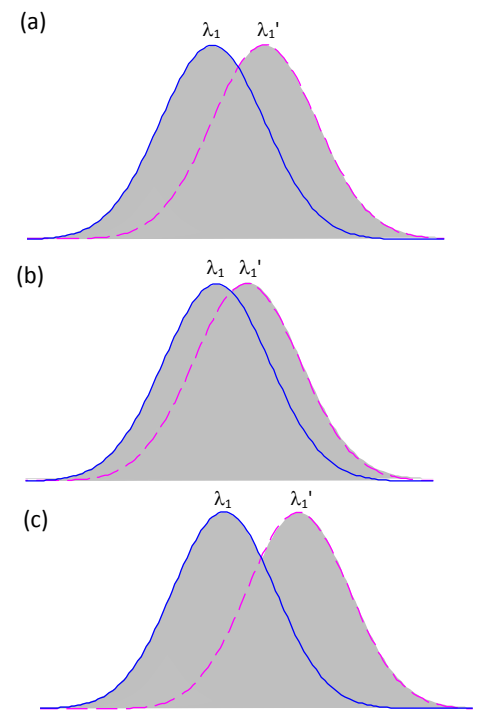

Figure 2. Optical power distribution between two FBGs (shaded area under the curves): (a) nominal position of peaks (no voltage applied); (b) and (c) two extreme positions of a voltage modulated

FBG peak, $\lambda_{1}$ ', with respect to the non-modulated peak, $\lambda_{1}$.

Although a temperature change will cause both peaks to spectrally shift due to the thermal strain and thermo-optic effect, the shift will be of the same magnitude and will not affect the reflected power distribution, allowing for temperature-independent strain/voltage measurements. The depth of the power modulation will depend on the sensor sensitivity to voltage and the FBG bandwidth. By careful sensor design, the FBG shift can be tailored so that a specific modulation depth is reached at the full-scale voltage measurement.

\section{INTERROGATION SYSTEM}

The proposed measurement system architecture is shown in Figure 3. A broadband source (BBS) is used to illuminate an array of FBG sensors, and the reflected signals are coupled off to a wavelength division multiplexing (WDM) filter having flat passband channel profiles, each channel being monitored by a photodetector (PD). Photodetector outputs may then be sampled using a data acquisition (DAQ) board for continual processing. 


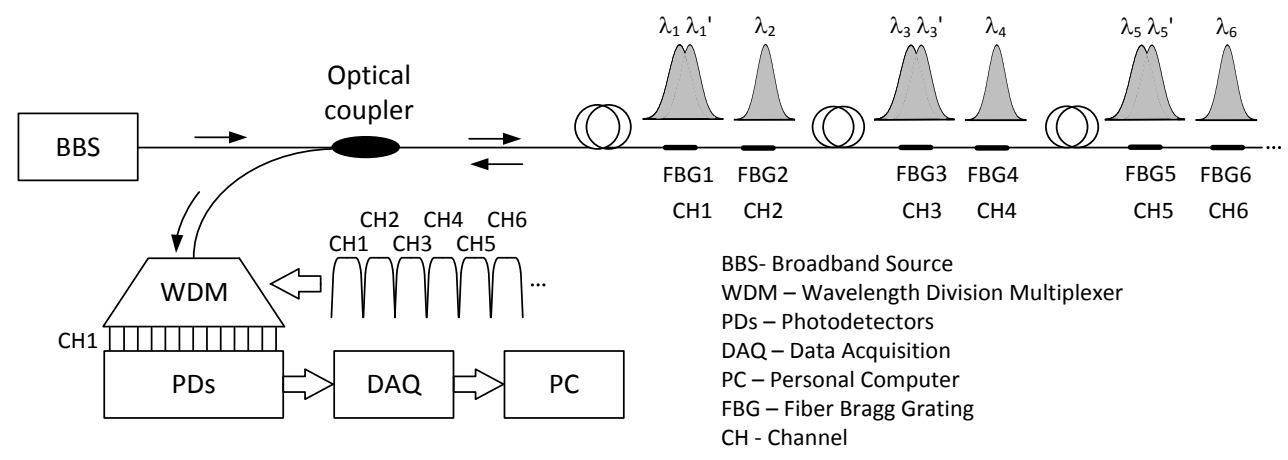

Figure 3. Functional block diagram of the proposed measurement system.

Since the proposed measurement technique relies on optical power modulation, any bend-induced attenuation would introduce voltage measurement errors via power fluctuations. To prevent this, the reflection from an additional FBG sensor positioned in close proximity to the voltage sensing transducer, i.e. $\lambda_{2}$ in Figure 3, may be used as a reference optical power, assuming that the local temperature change does not affect the power level reflected from the reference FBG. The voltage sensing and reference FBGs should be spectrally aligned with the centers of corresponding unique WDM channels. By combining a number of voltage and power reference sensors, the proposed measurement system may be utilized for distributed ac and dc voltage monitoring. Since the interrogation technique is parallel-processed and solid-state, the interrogation rate for each sensor is determined solely by the DAC/sampling rate at each photodetector and the signal processing capacity. The measurement resolution is determined by the photodetector and quantization noise.

\section{SOFTWARE SIMULATION}

A simulation program was written to theoretically evaluate the proposed measurement method. The program allowed for sweeping of the simulated FBG spectra over the required wavelength range, and the calculated total power levels were recorded in a data file. To simplify simulations, the following assumptions were made:

- the central wavelength of each sensor reflection spectrum falls centrally in each individual WDM channel;

- WDM channel bandwidths are wider than the maximum operating wavelength range of the sensors, and hence do not influence the reflected power from the sensors;

- WDM channel spacings are large enough to neglect crosstalk between channels;

Provided that the FBGs have normalized Gaussian profiles, the optical power reflected from the dual-peak FBG and detected by a photodetector is given by the integral of the FBG reflection functions according to the following equation:

$$
\mathrm{P}_{\mathrm{det}}(\lambda)=\int F B G(\lambda) d \lambda
$$

where $\operatorname{FBG}(\lambda)$ is the total reflection spectrum given by

$F B G(\lambda)=F B G 1(\lambda)+(1-F B G 1(\lambda)) \cdot F B G l^{\prime}(\lambda)$

and $F B G 1(\lambda)$ and $F B G 1^{\prime}(\lambda)$ are the FBGs reflection spectra approximated by

$$
\begin{aligned}
& F B G 1(\lambda)=\exp \left[\frac{-\left(\lambda-\lambda_{1}\right)^{2}}{2 \sigma_{F B G 1}^{2}}\right] \\
& F B G 1^{\prime}(\lambda)=\exp \left[\frac{-\left(\lambda-\lambda_{1}^{\prime}\right)^{2}}{2 \sigma_{F B G 1^{\prime}}^{2}}\right]
\end{aligned}
$$

In the above equations, $\lambda_{1}$ and $\lambda_{1}$ ' are the dual-peak wavelengths, and $\sigma_{F B G}$ and $\sigma_{F B G}$, determine the FBG bandwidths.

Representative examples of the simulation results are presented below:

Figure 4a shows the normalized optical power reflected from a dual-peak FBG as a function of the spacing between the $\lambda_{1}$ and $\lambda_{1}$ ' peaks. The dual-peak FBG was simulated as a combination of two FBGs having bandwidths of $0.4 \mathrm{~nm}$. For a certain wavelength range, the power-spacing relation is linear. The width of the linearity region is dependent on the FBGs' bandwidths and can be adjusted according to the requirements.

Figure $4 \mathrm{~b}$ shows the optical power modulation for the $\lambda_{1}$, peak subjected to sinusoidal wavelength changes with a peak wavelength amplitude of $100 \mathrm{pm}$. The signals are perfectly aligned. Thus, optical power modulation can be calibrated in terms of wavelength or strain/voltage. 
(a)

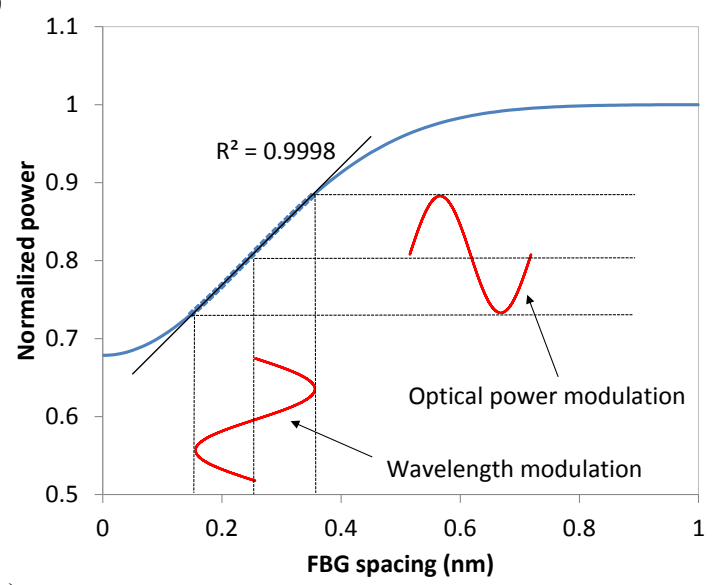

(b)

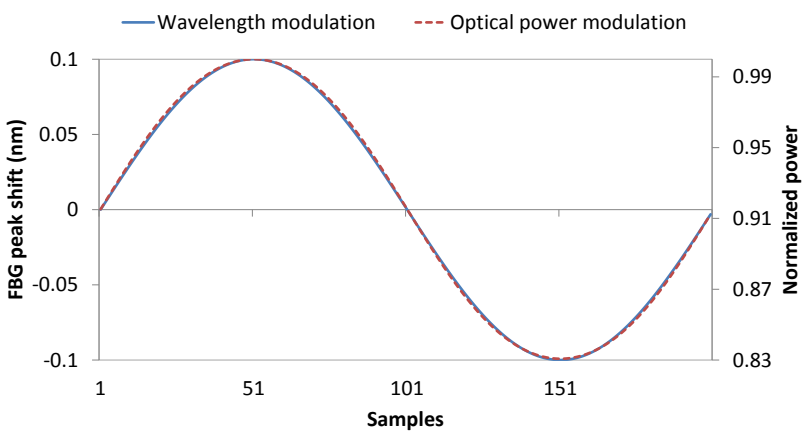

Figure 4. Normalized optical power levels versus FBG spacing for two gratings having equal reflections and bandwidths (a); Optical power modulation in response to FBG peak wavelength modulation within the linearity region (b).

\section{DISCUSSION}

Based on the simulation results presented in the previous section, a representative $5-\mathrm{kV}$ voltage sensor design may be proposed. In order to produce a dual-peak FBG wavelength modulation of $110 \mathrm{pm}$, a strain of around $88 \mu \varepsilon$ is required. Such a strain can be produced by a 7-mm long block of the K81 ceramic (a modified lead metaniobate) from Piezo Technologies [5], [6]. The K-81 material has a longitudinal piezoelectric charge constant $\mathrm{d}$ of $85 \mathrm{pm} / \mathrm{V}$ and a CTE of $1.3 \mathrm{ppm} /{ }^{\circ} \mathrm{C}$. At $5 \mathrm{kV}$ (rms) nominal voltage, the maximum voltage across the transducer is $7.07 \mathrm{kV}$ which translates to approximately $86 \mu \varepsilon$. For the sensor electrodes, invar may be used since this has a similar CTE of $1.2 \mathrm{ppm} /{ }^{\circ} \mathrm{C}$. A $7-\mathrm{mm}$ extended invar electrode would ensure equal shifts of both FBG peaks, facilitating temperature-independent optical power modulation. A 14-mm FBG sensor may be bonded to the composite electrode-piezoelectric structure with a suitable epoxy adhesive as discussed in Section II.

Optical components such as add-after-drop filters; cascaded Mach-Zehnder (CMZ) demultiplexers or WDM filters offering bespoke transmission profiles have been produced in siliconon-insulator (SOI) technology [10]-[12]. As the current silicon circuit technology allows for production of bespoke devices, a flat passband WDM filter with a channel bandwidth of $5 \mathrm{~nm}$ and the spacing between channels of $10 \mathrm{~nm}$ could be implemented for the proposed interrogation system with minimal physical size and the capability for low-cost mass production.

\section{CONCLUSIONS}

In this paper we have proposed a novel optical fiber voltage sensor comprising a piezoelectric transducer and fiber Bragg grating sensors. The transducer construction facilitates temperature and attenuation independent measurement of ac and dc voltage over a sensor array. The sensor design methodology is supported by software simulation, and a representative sensor design for voltage measurement up to 5 $\mathrm{kV}$ was presented. The interrogation of multiple sensors by a dedicated interrogation system presents great potential for smart grid applications where distributed voltage monitoring is required. Future work will concentrate on the laboratory evaluation of the proposed measurement method.

\section{REFERENCES}

[1] M. Di Bisceglie, C. Galdi, A. Vaccaro, D. Villacci, "Cooperative sensor networks for voltage quality monitoring in smart grids," IEEE PowerTech, 1-6 (2009)

[2] J. Luszcz, R. Smolenski, "Voltage harmonic distortion measurement issue in smart-grid distribution system," APEMC, 841-844 (2012)

[3] D.M. Parker, N.D. McCollough, "Medium-voltage sensors for the smart grid: Lessons learned," IEEE PES GM, 1-7 (2011)

[4] P. Kansal, A. Bose, "Smart grid communication requirements for the high voltage power system," IEEE PES GM, 1-6 (2011)

[5] P. Niewczas, L. Dziuda, G. Fusiek, J. R. McDonald, "Design and Evaluation of a Pre-Prototype Hybrid Fiber-Optic Voltage Sensor for a Remotely Interrogated Condition Monitoring System," IEEE Transactions on Instrumentation and Measurement 54(4), 1560-1564 (2005)

[6] L. Dziuda, P. Niewczas, G. Fusiek, J. R. McDonald, "Hybrid Fiber-Optic Voltage Sensor for Remote Monitoring of Electrical Submersible Pump Motors," Optical Engineering 44(6), 644011-6 (2005)

[7] G. Fusiek, P. Niewczas, J. R. McDonald, "Feasibility Study of the Application of Optical Voltage and Current Sensors and an Arrayed Waveguide Grating for Aero-Electrical Systems," Sensors and Actuators: A. Physical 147, 177-182 (2008)

[8] P. Orr, G. Fusiek, C.D. Booth, P. Niewczas, A. Dysko, F. Kawano, P. Beaumont, T. Nishida, "Flexible protection architectures using distributed optical sensors," IET DSPS, 155 (2012)

[9] Y. J. Rao, "In Fibre Bragg grating sensors," Meas. Sc. Tech. 8, 355-375, (1997)

[10] F. Horst, W.M.J. Green, S. Assefa, S.M. Shank, B.J. Offrein, Y.A. Vlasov, "WDM Filters For Silicon Photonics Transceivers," OECC, 842-844 (2011)

[11] D. Feng, N. Feng, H. Liang, W. Qian, C. Kung, J. Fong, and M. Asghari, "Monolithic WDM Filter in Silicon-on-Insulator for Diplexer / Triplexer Application," Integrated Photonics and Nanophotonics Research and Applications, OSA, ITuA4 (2008)

[12] B. J. Offrein, G. L. Bona, F. Horst, W. M. Salemink, R. Beyeler, and R. Germann, "Wavelength tunable optical add-after-drop filter with flat passband for WDM networks," IEEE Photonics Technology Letters 11(2), 239-241 (1999) 